\title{
Sensitivities of future reactor and long-baseline neutrino experiments to NSI
}

\author{
Pouya Bakhti® and Meshkat Rajaee \\ Institute for research in fundamental sciences (IPM), PO Box 19395-5531, Tehran, Iran
}

(Received 4 November 2020; accepted 19 March 2021; published 6 April 2021)

\begin{abstract}
We investigate the potential of future data from next generation long-baseline neutrino experiments, DUNE and T2HK and the upcoming reactor experiment JUNO in constraining neutral current nonstandard neutrino interactions (NSI) parameters. JUNO is going to provide the most precise measurements of solar neutrino oscillation parameters as well as determining the neutrino mass ordering. We study how the results of JUNO combined with those of long-baseline neutrino experiments such as DUNE and T2HK can help to determine oscillation parameters and constrain NSI parameters. We present excluded regions in NSI parameter space, $\epsilon_{\alpha \beta}$ assuming Standard Model (SM) as the null hypothesis. We further explore the correlations between the NSI parameters and the $C P$-violation phase.
\end{abstract}

DOI: $10.1103 /$ PhysRevD.103.075003

\section{INTRODUCTION}

Neutrino oscillations can successfully describe neutrino flavor transitions. In 1978, the matter potential for neutrino propagation through matter was proposed [1]. Wolfenstein introduced nonstandard interactions to explain neutrino oscillations with massless neutrinos [1].

The nonstandard neutrino Interactions (NSI) appear in many new physics models where the neutrino interactions with ordinary matter are parametrized at low energy in terms of effective flavor-dependent couplings $\epsilon_{\alpha \beta}$. Several models of physics beyond the Standard Model (SM) predict NSI. For instance, NSI arise naturally in many neutrino mass models trying to explain the smallness of neutrino mass [2-8] and the large neutrino mixing angles [9-11]. Thus, it is crucial to understand how the presence of NSI can affect the standard neutrino oscillation in the matter. The presence of NSI couplings can affect neutrino production, detection, and propagation. Such a new interaction leads to a rich phenomenology and has been extensively studied in the literature. NSI were proposed as a solution to the solar neutrino problem [12]. Also, see Refs. [13-18] for studies of their impact on solar neutrinos oscillations. NSI impacts on accelerator neutrinos and atmospheric neutrinos have been explored in [19-48]

NSI can be formulized as a $d=6$ four-fermion effective operators involving neutrino fields given in two main categories: Operators affecting charged-current (CC) neutrino interactions $\left(\bar{l}_{\alpha} \gamma_{\mu} P_{L} \nu_{\beta}\right)\left(\bar{q} \gamma^{\mu} P q^{\prime}\right)$, where $l$ stands for a

Published by the American Physical Society under the terms of the Creative Commons Attribution 4.0 International license. Further distribution of this work must maintain attribution to the author(s) and the published article's title, journal citation, and DOI. Funded by SCOAP. charged lepton, $P$ stands for one of the chirality projectors $P_{R, L} \equiv \frac{1}{2}\left(1 \pm \gamma_{5}\right), \alpha$ and $\beta$ are lepton flavor indices, and $q$ and $q^{\prime}$ represent quarks, or operators affecting neutralcurrent (NC) neutrino interactions which are given in the form $\left(\bar{\nu}_{\alpha} \gamma_{\mu} P_{L} \nu_{\beta}\right)\left(\bar{f} \gamma^{\mu} P f\right)$, where $f$ represents SM fermion. Charged-current neutrino interactions NSI affect neutrino production and detection processes. See Refs. [49-59] for the studies on the potential of neutrino oscillation experiments to study NSI affecting neutrino production and detection. On the other hand, NC NSI affects neutrino propagation and depending on the detection process, might also affect neutrino detection. Since the present bounds on the CC NSI parameters are strong while the constraints on NC NSI are not very stringent, in this paper we focus on the neutral-current NSI.

Interestingly, matter effects are negligible for the reactor experiments due to their low energy and relatively shortbaseline. This also holds for NSI effects during propagation of neutrinos and therefore, we can only assume the NSI effects at sources and detectors. Using this characteristic of reactor experiments, we focus on investigating how well, the future long-baseline neutrino experiments can constrain NSI. The exact value of the mixing angle $\theta_{13}$ has now been determined to good accuracy by reactor experiments such as Daya Bay [60] and RENO [61] with the previous hint from the combination of KamLAND and solar neutrino observatories data [62]. These experiments provided evidence for a nonzero $\theta_{13}$, with $5.2 \sigma$ and $4.9 \sigma$ results respectively and their results show that $\theta_{13}$ is very close to $8.4^{\circ}$. Besides, the $\mathrm{T} 2 \mathrm{~K}$ experiment has also confirmed the nonzero and moderately large value of $\theta_{13}$ in the standard three-flavor oscillation scenario [63]. The future reactor experiment Jiangmen Underground Neutrino Observatory (JUNO) is going to determine the mass ordering and is 
going to measure $\Delta m_{21}^{2}$ and $\theta_{12}$ to the percent level [64]. JUNO is a multipurpose neutrino experiment designed to detect solar neutrinos and geo-neutrinos in addition to determining neutrino mass hierarchy and precisely measurement of oscillation parameters.

The long-baseline Deep Underground Neutrino Experiment (DUNE) with the main goal of searching for leptonic $C P$-violation phase and testing the three massive neutrinos paradigm is going to provide precision measurements of oscillation probabilities that can be used to probe NC NSI. The effects of NSI and how they modify neutrino propagation in the DUNE is studied in [65]. The capability of the DUNE near detector to constrain NSI describing the production of neutrinos is studied in $[49,66]$. The Tokai to Hyper-Kamiokande (T2HK) experiment uses an upgraded J-PARC beam with a detector located $295 \mathrm{~km}$ away from the source with a spectrum peaked at $0.6 \mathrm{GeV}$ [67]. The effect of the presence of NSI in DUNE, T2HK and T2HKK has been studied in [68]. References [69,70] present the Daya Bay and T2K results on NSI.

In this paper, we study how combining data of future long-baseline experiments DUNE and T2HK with the precise measurements of future reactor experiment JUNO is going to determine the standard oscillation parameters by a higher accuracy. Moreover, we present the constraints on NSI parameter space using these future neutrino experiments. In more details, we study how well the precise measurements of oscillation probabilities at DUNE, T2HK and JUNO can be used to probe the existence of nonstandard neutrino neutral current interactions and discuss how using the combined data of the upcoming DUNE, T2HK and JUNO experiments can constrain NSI assuming it is consistent with the standard paradigm. We will investigate the correlations between the NSI parameters and the $C P$-violation phase using our simulations for these future neutrino experiments.

The paper is organized as follows. In Sec. II, we discuss the formalism. In Sec. III, we discuss the details of our simulation and the experiments. In Sec. IV, we present our results. Our conclusions will be presented in Sec. V.

\section{FORMALISM}

In this section, we briefly discuss how the presence of NSI modifies the effective Hamiltonian for neutrino propagation in the matter. The total Hamiltonian in the flavor basis is given by [71]

$$
\begin{aligned}
H= & H_{\mathrm{vac}}+H_{\text {matt }} \\
= & \frac{1}{2 E}\left(U\left[\begin{array}{ccc}
m_{1}^{2} & 0 & 0 \\
0 & m_{2}^{2} & 0 \\
0 & 0 & m_{3}^{2}
\end{array}\right] U^{\dagger}\right. \\
& \left.+a\left[\begin{array}{ccc}
1+\varepsilon_{e e} & \varepsilon_{e \mu} & \varepsilon_{e \tau} \\
\varepsilon_{e \mu}^{*} & \varepsilon_{\mu \mu} & \varepsilon_{\mu \tau} \\
\varepsilon_{e \tau}^{*} & \varepsilon_{\mu \tau}^{*} & \varepsilon_{\tau \tau}
\end{array}\right]\right)
\end{aligned}
$$

where $E$ is the neutrino energy and $a$ is the matter-effect parameter and is given by

$a=2 \sqrt{2} G_{F} N_{e} E=7.6324 \times 10^{-5}\left(\mathrm{eV}^{2}\right)\left(\frac{\rho}{\mathrm{g} / \mathrm{cm}^{3}}\right)\left(\frac{E}{\mathrm{GeV}}\right)$,

and the " +1 " term in Eq. (1) corresponds to the standard contribution, and

$$
\epsilon_{\alpha \beta}=\sum_{f=e, u, d} \frac{N_{f}(x)}{N_{e}(x)} \epsilon_{\alpha \beta}^{f}=\left[4+3 Y_{n}(x)\right] \epsilon_{\alpha \beta}^{f},
$$

with

$$
Y_{n}(x) \equiv \frac{N_{n}(x)}{N_{e}(x)}
$$

describes the nonstandard part and is written in terms of the effective couplings of protons $(p)$ and neutrons $(n)$. We have assumed $\epsilon_{\alpha \beta}^{e}=\epsilon_{\alpha \beta}^{u}=\epsilon_{\alpha \beta}^{d}=\epsilon_{\alpha \beta}^{f}$ and $N_{e}(x)=$ $N_{p}(x)=N_{n}(x)$ so $Y_{n}(x)=1$.

$U$ is the Pontecorvo-Maki-Nakagawa-Sakata (PMNS) matrix and is parametrized as

$$
U=\left(\begin{array}{ccc}
c_{12} c_{13} & s_{12} c_{13} & s_{13} e^{-i \delta_{C P}} \\
-s_{12} c_{23}-c_{12} s_{13} s_{23} e^{i \delta_{C P}} & c_{12} c_{23}-s_{12} s_{13} s_{23} e^{i \delta_{C P}} & c_{13} s_{23} \\
s_{12} s_{23}-c_{12} s_{13} c_{23} e^{i \delta_{C P}} & -c_{12} s_{23}-s_{12} s_{13} c_{23} e^{i \delta_{C P}} & c_{13} c_{23}
\end{array}\right),
$$

where $s_{i j}$ and $c_{i j}$ denote the sine and cosine of the mixing angle $\theta_{i j}$, and $\delta_{C P}$ is the (Dirac) $C P$ phase [71,72]. The strongest bounds on NSI parameters in propagation come from the global fit to neutrino oscillation data in Ref. [73]. In presence of NSI in propagation, global analysis of neutrino oscillation data are compatible with two solutions: the LMA solution and the LMA-dark solution. In this work, we focus on the LMA solution. Since we assume the same NSI couplings for up and down quarks, LMA-dark solution is excluded by COHERENT results [74] at about $3 \sigma$ C.L.; 
However, considering a general coupling of NSI with quarks where $\epsilon_{\alpha \beta}^{u} \neq \epsilon_{\alpha \beta}^{d}$, the LMA-D region remains allowed at the $3 \sigma$ C.L. level [75].

The oscillation probability in the presence of NSI is given by [76]

$$
P_{\nu_{\alpha} \rightarrow \nu_{\beta}}=\left|\left\langle\nu_{\beta}\left|e^{-i H L}\right| \nu_{\alpha}\right\rangle\right|^{2} .
$$

Since reactor experiments are sensitive to $P\left(\bar{\nu}_{e} \rightarrow \bar{\nu}_{e}\right)$ and long-baseline experiments are sensitive to $P\left(\stackrel{(-)}{\nu}_{\mu} \rightarrow\right.$ $\left.\stackrel{(-)}{\nu}_{e}\right)$ and $P\left(\stackrel{(-)}{\nu}_{\mu} \rightarrow \stackrel{(-)}{\nu}_{\mu}\right)$, in order to study the impact of nonstandard interactions on these experiments, we only need to focus on these oscillation probabilities. Since the oscillation probability $P\left(\nu_{\mu} \rightarrow \nu_{e}\right)$ does not have strong dependence on $\left|\epsilon_{\mu \tau}\right|$ and $\left|\epsilon_{\mu \mu}-\epsilon_{\tau \tau}\right|$ and there are already strong bounds on $\left|\epsilon_{\mu \tau}\right|$ and on $\left|\epsilon_{\mu \mu}-\epsilon_{\tau \tau}\right|$ [73], we take $\epsilon_{e \mu}$, $\epsilon_{e \tau}$ and $\epsilon_{e e}-\epsilon_{\mu \mu}$ nonzero. To perform our analysis, we set true values of standard oscillation parameters from nu-fit [77] and the uncertainties from [73]. Also, we set the true values of $\epsilon_{e \mu}, \epsilon_{e \tau}$ and $\epsilon_{e e}-\epsilon_{\mu \mu}$ to zero.

\section{DETAILS OF THE ANALYSIS}

In this section, we discuss the approach of our analysis in obtaining the results presented in this paper. For the statistical inferences, we simulate 7 years of data collection for DUNE experiment, 6 years for JUNO experiment and 10 years of data collection for T2HK experiment.

We have considered that DUNE consists of a 40 kiloton liquid argon detector and utilizes a 1.2 MW proton beam to produce neutrino and antineutrino beams from in-flight pion decay, originating $1300 \mathrm{~km}$ upstream at Fermilab. The neutrino energy ranges between 0.25 and $8 \mathrm{GeV}$ and the flux peak occurs around $3.0 \mathrm{GeV}$. All the details of the DUNE experiment are taken from $[49,78]$. We have

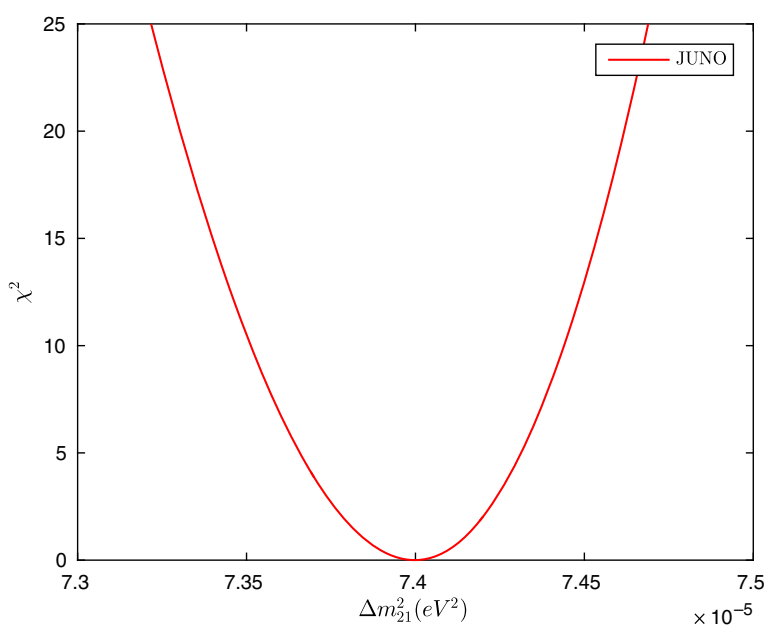

considered the energy resolutions for detection as $15 \% / \sqrt{E(\mathrm{GeV})}$ for $\nu_{e}$ and $20 \% / \sqrt{E(\mathrm{GeV})}$ for $\nu_{\mu}$. Also, we have considered the efficiency of detection as $80 \%$ and $85 \%$ for $\nu_{e}$ and $\nu_{\mu}$, respectively. We have set the calibration error as $2 \%$ and the flux uncertainty is taken to be $5 \%$. For our analysis we consider the energy range between $0.25 \mathrm{GeV}$ to $8 \mathrm{GeV}$ devided into 31 equal sized bins in the unit of $0.25 \mathrm{GeV}$.

For the T2HK experiment, we have considered the mass of $374 \mathrm{kt}$ for the water Cherenkov detector [79]. T2HK experiment uses an upgraded $30 \mathrm{GeV}$ J-PARC beam with a power of 1.3 MW and its detector is located $295 \mathrm{~km}$ away from the source. All the details of the T2HK experiment can be found in [79-81]. We have taken the energy resolutions for detection as $8.5 \% / \sqrt{E(\mathrm{GeV})}$ for $\nu_{e}$ and $\nu_{\mu}$. The efficiency of detection is set as $50 \%$ for $\nu_{e}$ and $90 \%$ for $\nu_{\mu}$, respectively. The calibration error is set to $0.1 \%$ and the flux uncertainty is taken to be $2.5 \%$. We have considered the energy range between $0.4 \mathrm{GeV}$ to $1.2 \mathrm{GeV}$ divided into 20 equal-sized bins.

Medium-baseline reactor experiment JUNO consists of nuclear plant reactor complex at Yangjiang and Taishan with a power of $36 \mathrm{GW}$ and a $20 \mathrm{kt}$ scintillator detector with a baseline of $52 \mathrm{~km}$. We have considered all the details of JUNO the same as given in $[82,83]$ and we have assumed 6 years of data taking for JUNO. The energy resolutions for detection is taken to be $3 \% / \sqrt{E(\mathrm{GeV})}$ for $\nu_{e}$ and $\nu_{\mu}$. The efficiency of detection is set as $100 \%$ for $\nu_{e}$. We have set the calibration error as $3 \%$ and the flux uncertainty of $5 \%$. For our analysis, we consider the energy range between $1.8 \mathrm{MeV}$ to $8 \mathrm{MeV}$ divided into 62 equal sized bins.

For our analysis, we have considered the value of $\theta_{13}$ as measured by the Daya Bay experiment. As mentioned in the previous section, we set true values of standard oscillation parameters from nu-fit [77] and the uncertainties from [73] while setting the true values of $\epsilon_{e \mu}, \epsilon_{e \tau}$ and

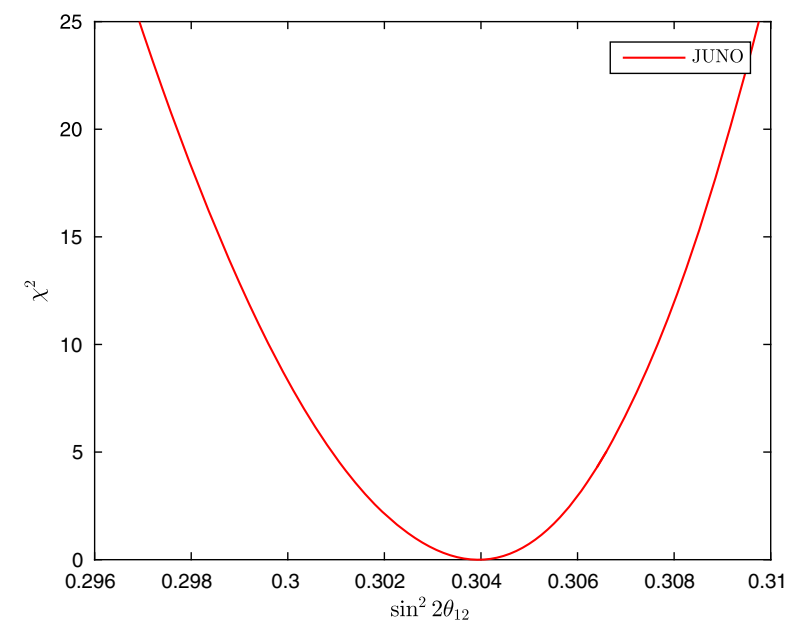

FIG. 1. Chi-squared vs. $\Delta m_{21}^{2}$ and $\sin ^{2} \theta_{12}$ assuming six years of data taking for the JUNO experiment. We have set the true values from nu-fit [77]. 
$\epsilon_{e e}-\epsilon_{\mu \mu}$ equal to zero and their uncertainties as $0.1,0.25$ and 0.3 , respectively [73]. We marginalize over NSI phases of $\phi_{e \mu}, \phi_{e \tau}$ and other systematic using the so-called pull method. In the following, we will explore the future sensitivity of the combination of DUNE, T2HK and JUNO to determine standard neutrino oscillation parameters in the presence of NSI and try to constrain NSI parameters. For the statistical inferences we have considered Asimov data set approximation, and we have assumed the standard model as the true model. We have used the chisquared method with the assumption of Gaussian distribution of errors. In our simulation, we used GLoBES software $[84,85]$ and include the NSI from Ref. [76]. Let us discuss the main results of the effects of NC NSIs on the determination of the standard oscillation parameters considering JUNO, DUNE and T2HK experiments.

\section{RESULTS}

In this section, we discuss our results which are shown in Figs $1-8$. Figure 1 shows the sensitivity of JUNO to the measurement of solar neutrino oscillation parameters $\Delta m_{21}^{2}$ and $\theta_{12}$. We have assumed 7 years of data taking for DUNE experiment, 6 years for JUNO experiment and 10 years for T2HK experiment. We have set the true values of standard oscillation parameters from nu-fit [77] and the uncertainties from [73]. We have also assumed $\epsilon_{e \mu}=\epsilon_{e \tau}=$ $\epsilon_{e e}-\epsilon_{\mu \mu}=0$. As it is expected, NSI does not affect JUNO
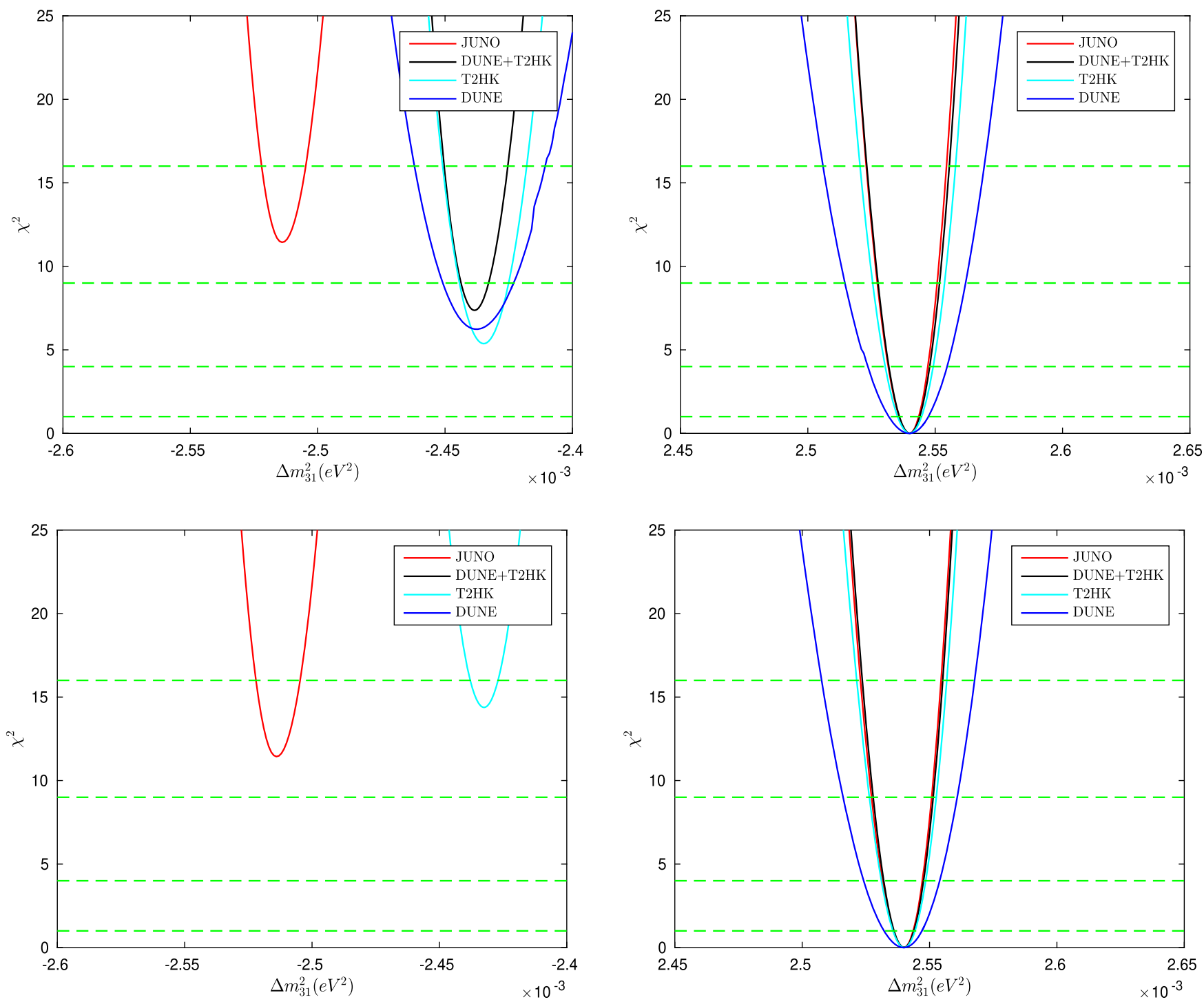

FIG. 2. Chi-squared vs. $\Delta m_{31}^{2}$ assuming normal ordering as the true ordering. Red, black, cyan and blue curves show chi-squared vs. $\Delta m_{31}^{2}$ for JUNO, DUNE + T2HK, T2HK, and DUNE, respectively. We have assumed 7 years of data taking for DUNE, 6 years for JUNO and 10 years for T2HK. The green dashed lines show $1 \sigma, 2 \sigma, 3 \sigma$ and $4 \sigma$ lines. Upper (lower) panels are plotted in the presence (absence) of NSI. 

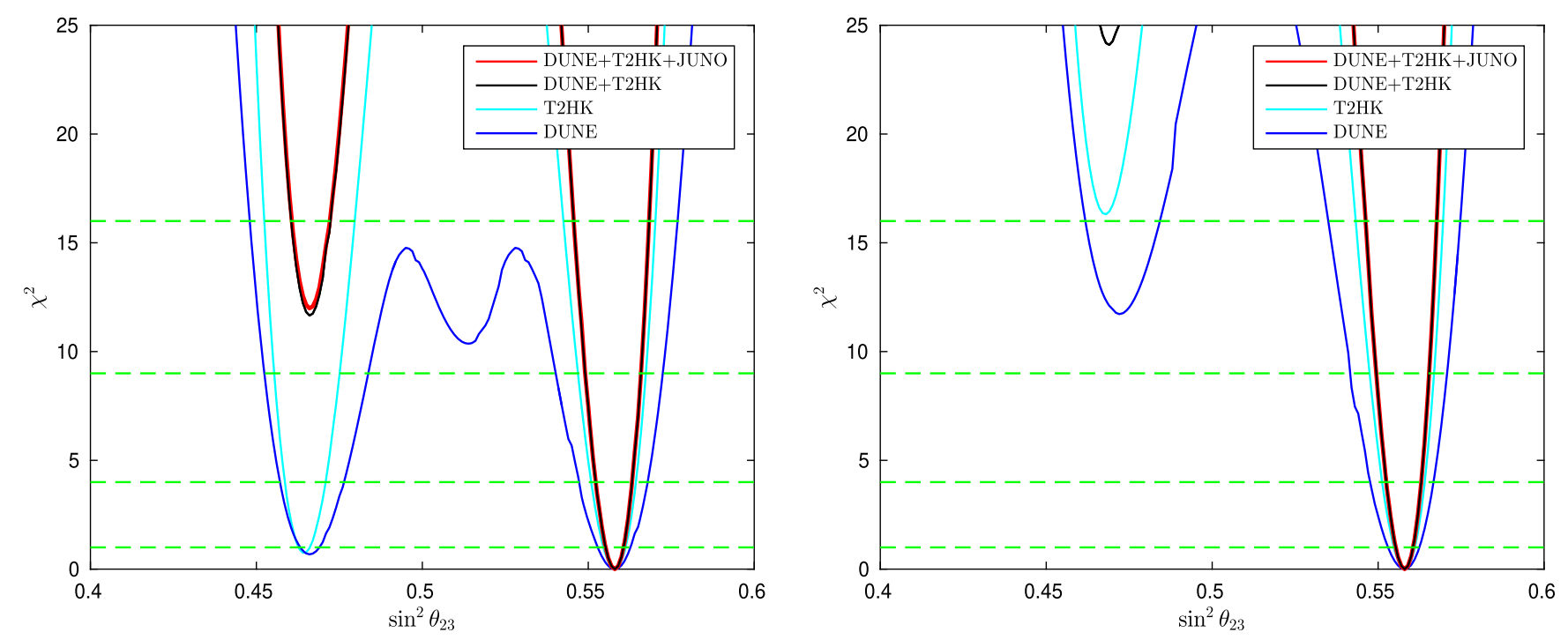

FIG. 3. Chi-squared vs. $\sin ^{2} \theta_{23}$. The black, blue, and red curves indicate T2HK and DUNE and the combination of T2HK, DUNE, and JUNO, assuming $\sin ^{2} \theta_{23}=0.56$ [77]. The left (right) panel shows the sensitivity of DUNE, T2HK, DUNE + T2HK, and DUNE + $\mathrm{T} 2 \mathrm{HK}+\mathrm{JUNO}$ to the determination of the octant of $\theta_{23}$ in the presence (absence) of NSI.

measurements on the oscillation parameters as studied in [18]. Thus, in the presence of NSI, JUNO can measure these oscillation parameters with high precision.

Using the characteristics of DUNE, T2HK, and JUNO, we show the results for the analysis of measurement of $\Delta m_{31}^{2}$ in the presence of NSI. Figure 2 indicates chi-squared vs. $\Delta m_{31}^{2}$ for JUNO, T2HK, DUNE, and DUNE + T2HK, assuming normal ordering (NO) as the true ordering. As can be seen from the figure, $\Delta m_{31}^{2}<0$ is excluded at $2 \sigma$ by DUNE, T2HK and their combination and at more than $3 \sigma$ by JUNO. As it can be seen in Fig. 2(b), JUNO can determine $\Delta m_{31}^{2}>0$ better than DUNE, T2HK and the

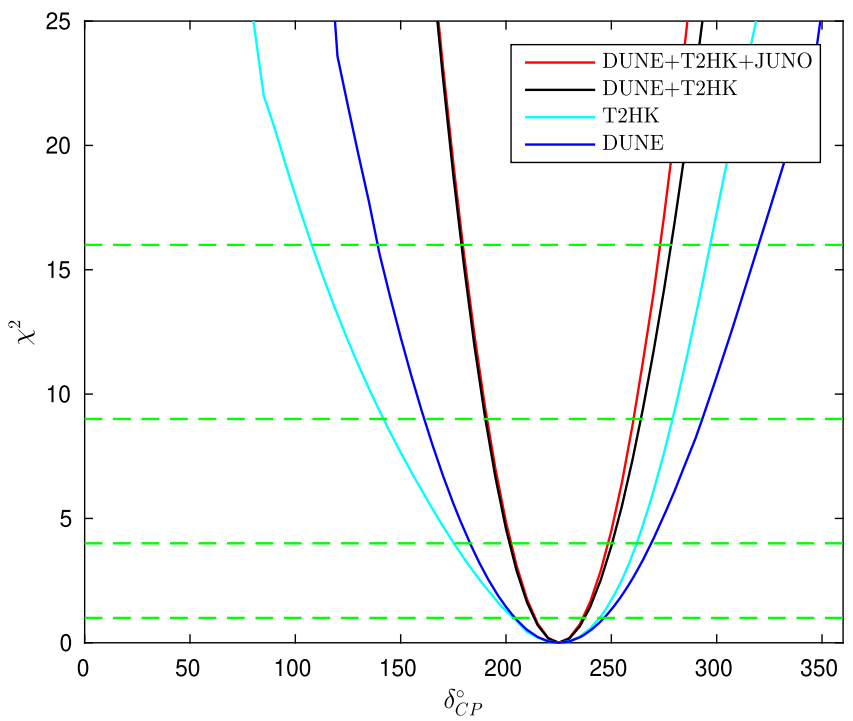

combination of DUNE and T2HK. Notice that considering only standard interaction, DUNE and T2HK can determine mass ordering more precisely than JUNO. T2HK, and DUNE can exclude the wrong mass ordering at $4 \sigma$ and $5 \sigma$ C.L., respectively.

Due to the importance of the $C P$-violation phase and determination of the octant of $\theta_{23}$, we study how these future experiments can determine these parameters. Figure 3 shows the sensitivity of these experiments to the determination of $\theta_{23}$. We have set the true value of $\sin ^{2} \theta_{23}=0.56$ as given in [77]. In the left panel, we have marginalized over NSI uncertainties and in the right panel,

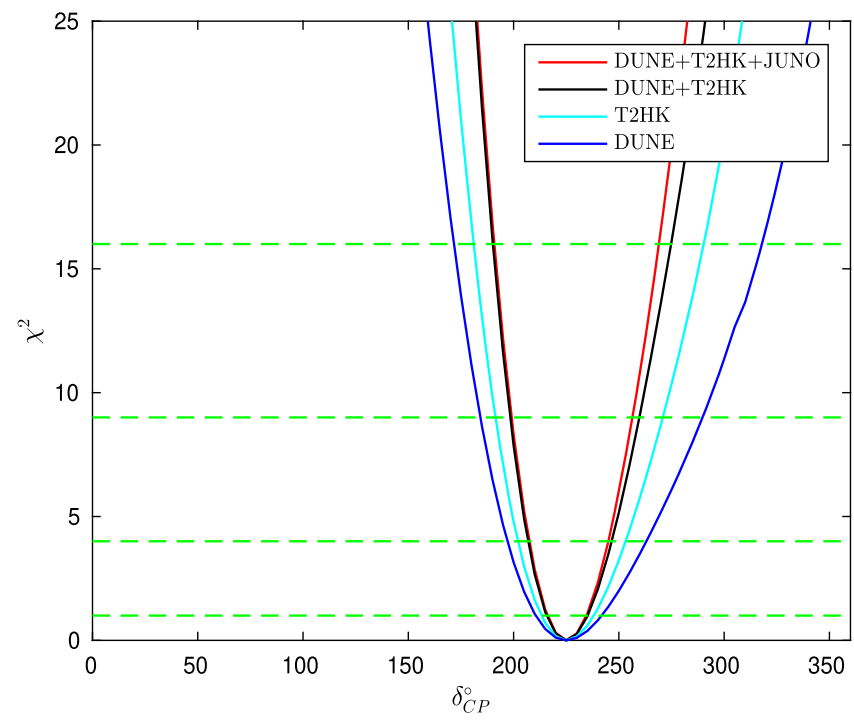

FIG. 4. Chi-squared vs. $\delta_{C P}$ assuming 7 years of data taking for DUNE, 6 years for JUNO and 10 years for T2HK in the presence of NSI (right) and in the absence of NSI (left). The red, black, blue and the cyan curves corresponds to the combination of T2HK, DUNE, and JUNO, the combination of T2HK and DUNE, DUNE, and T2HK. We have assumed $\delta_{C P}=225^{\circ}[77]$. 
we have set NSI parameters to zero. As can be seen from the plot, in the presence of NSI, DUNE and T2HK cannot determine the octant independently. Combining DUNE and T2HK data can determine the octant of $\theta_{23}$ as demonstrated. Although adding JUNO can improve the sensitivity of the determination of the octant (one unit of chi-squared), the combination of these experiments excludes the wrong octant at $3 \sigma$ C.L.. When NSI is off, DUNE, T2HK and the combination of DUNE and T2HK can exclude the wrong octant at $3 \sigma, 4 \sigma$ and $5 \sigma$ C.L., respectively.

Fig. 4 indicates chi-squared vs. $\delta_{C P}$ for T2HK, DUNE, the combination of T2HK and DUNE and the combination of all three experiments, assuming $\delta_{C P}=225^{\circ}$ as the true value [77] in the presence and the absence of NSI. As can be seen in the left panel, in the presence of NSI, T2HK, and
DUNE can exclude no $C P$-violation case at more than $2 \sigma$. Combining T2HK, DUNE, and JUNO data can exclude the no $C P$-violation case at more than $4 \sigma$ C.L.. In the absence of NSI, T2HK, and DUNE can exclude no $C P$-violation case at more than $3 \sigma$ and the combination of T2HK, DUNE, and JUNO data can exclude the no $C P$-violation case at more than $5 \sigma$ C.L.

Since the matter effect is more important for DUNE than for T2HK, (because of the larger energy and the longer baseline of DUNE), DUNE is more sensitive to the determination of the NSI parameters. We assume that the data are consistent with the SM and the NO, and plot the chi-squared vs. $\epsilon_{e \mu}, \epsilon_{e \mu}$, and $\epsilon_{e e}-\epsilon_{\mu \mu}$ for the following four cases: considering T2HK data, considering DUNE data, considering the combination of DUNE and T2HK

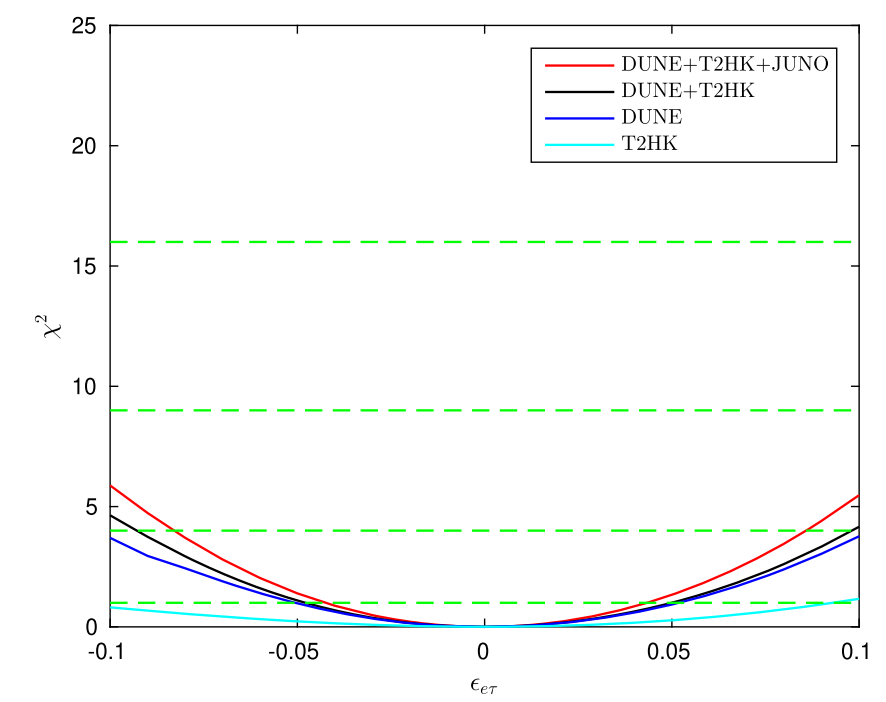

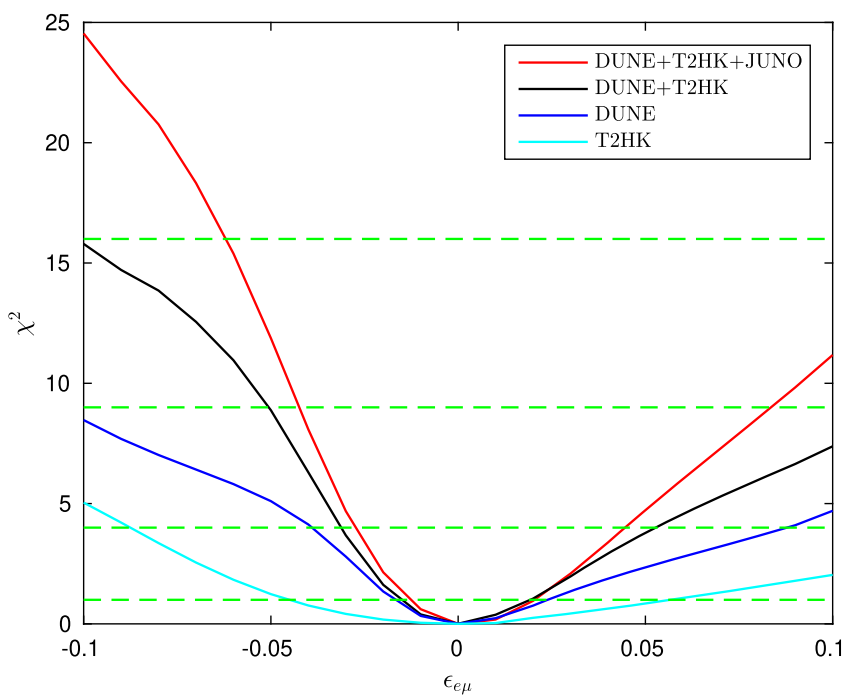

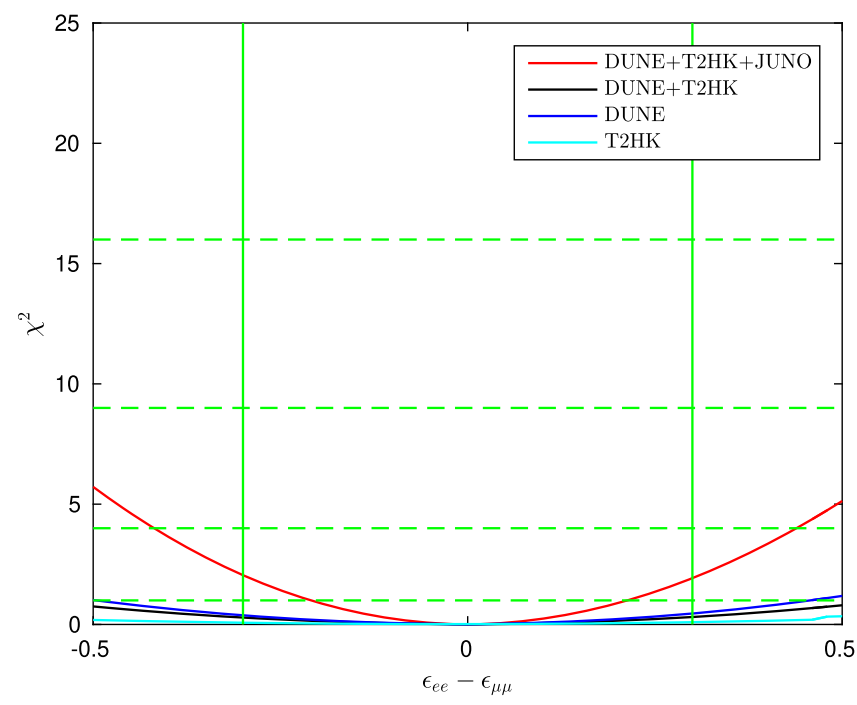

FIG. 5. Chi-squared vs. $\epsilon_{e \mu}, \epsilon_{e \tau}$ and $\epsilon_{e e}-\epsilon_{\mu \mu}$. The red, black, blue, and cyan curves correspond to the combined data of all experiments, the combination of T2HK and DUNE, DUNE and T2HK, respectively. The green vertical lines indicate the current constraints at $1 \sigma$ C.L. [73]. The green dashed horizontal lines show the $1 \sigma, 2 \sigma, 3 \sigma$, and $4 \sigma$ region. 
data and considering the combined data of T2HK, DUNE and JUNO. The results are shown in Fig. 5. As it can be seen from the figure, DUNE is more sensitive to the measurement of $\epsilon_{e \mu}$ and $\epsilon_{e \tau}$ than T2HK. However, considering the combination of T2HK, DUNE and JUNO data can increase the accuracy to determine $\epsilon_{e \mu}$ and $\epsilon_{e \tau}$. Since JUNO reduces the uncertainties of the oscillation parameters, this combination works better. As indicated in the lower panel of Fig. 5, DUNE and T2HK cannot determine $\epsilon_{e e}-\epsilon_{\mu \mu}$. Moreover, the combined analysis of DUNE and T2HK data shows that their combination is not sensitive to the determination of $\epsilon_{e e}-\epsilon_{\mu \mu}$. However, combining the data of DUNE and T2HK experiments while including JUNO data, is interestingly sensitive to the determination of this parameter. Notice that as mentioned before, the current constraints on $\epsilon_{e \mu}, \epsilon_{e \tau}$ and $\epsilon_{e e}-\epsilon_{\mu \mu}$ at $1 \sigma$ C.L. are $0.1,0.25$ and 0.3 , respectively [73]. The vertical green lines indicate the current constraints. DUNE can constrain $\epsilon_{e \mu}$ independently more stringent than the current constraints. Combining the data of DUNE, T2HK with those of JUNO can determine $\epsilon_{e \mu}, \epsilon_{e \tau}$, and $\epsilon_{e e}-\epsilon_{\mu \mu}$ more stringent than the current constraints.

We show the results for the analysis of the simultaneous measurements of $\epsilon_{e \mu}$ and $\delta_{C P}$ in Fig. 6, considering DUNE, T2HK, the combination of T2HK and DUNE and considering the combination of DUNE, T2HK, and JUNO. Although DUNE and T2HK can constrain $\delta_{C P}$, they do not have a strong sensitivity to $\epsilon_{e \mu}$. As demonstrated, combining T2HK, DUNE and JUNO can increase the sensitivity of the simultaneous measurement of $\epsilon_{e \mu}$ and $\delta_{C P}$.
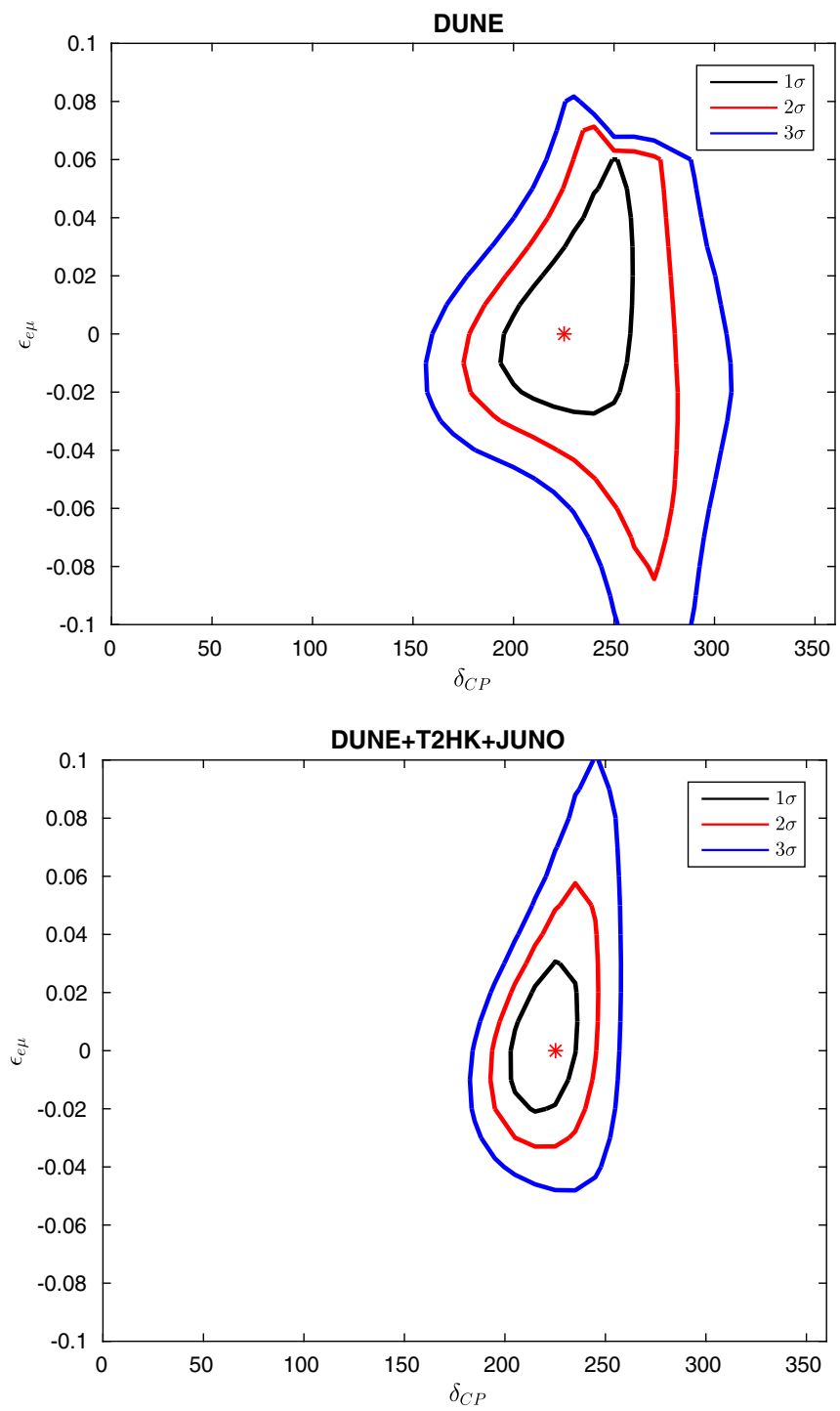

FIG. 6. $\epsilon_{e \mu}$ vs. $\delta_{C P}$ plotted for T2HK, DUNE, the combination of DUNE and T2HK and the combination of T2HK, DUNE and JUNO experiments. The red star shows the assumed true value for $\delta_{C P}$ and $\epsilon_{e \mu}$. We have assumed 7 years of data taking for DUNE, 6 years of data taking for JUNO and 10 years of data taking for T2HK. 

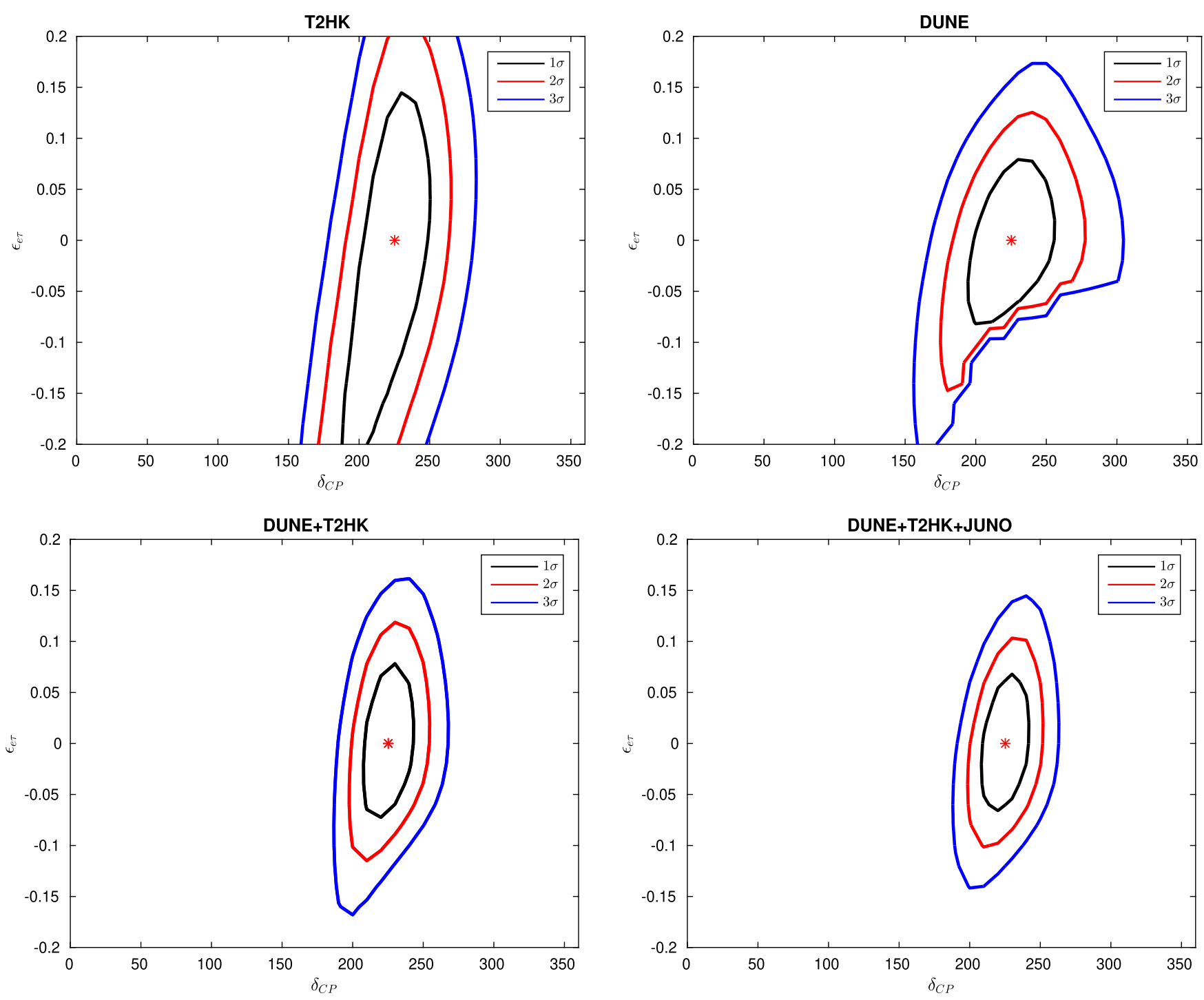

FIG. 7. $\epsilon_{e \tau}$ vs. $\delta_{C P}$ plotted for T2HK, DUNE, T2HK + DUNE, and T2HK + DUNE + JUNO. The red star shows the assumed true value for $\delta_{C P}$ and $\epsilon_{e \tau}$. We have assumed 7 years of data taking for DUNE, 6 years of data taking for JUNO and 10 years of data taking for $\mathrm{T} 2 \mathrm{HK}$.

In more detail, including JUNO improves $\delta_{C P}$ slightly while improving strongly the determination of $\epsilon_{e \mu}$.

Figure 7 shows the results of combining T2HK, DUNE, and JUNO to measure simultaneously $\epsilon_{e \tau}$ and $\delta_{C P}$. In the same way, the combination of data of these experiments can increase the accuracy of the simultaneous measurements of $\epsilon_{e \tau}$ and $\delta_{C P}$.

Finally, we discuss simultaneous measurement of $\epsilon_{e e}-\epsilon_{\mu \mu}$ and $\delta_{C P}$ for T2HK, DUNE, the combination of T2HK and DUNE, and the combination of DUNE, T2HK, and JUNO in Fig. 8. Although DUNE and T2HK are not sensitive to the simultaneous measurement of $\epsilon_{e e}-\epsilon_{\mu \mu}$ independently, combining T2HK, DUNE, and JUNO has a strong sensitivity to it and can increase the sensitivity to the determination of $\delta_{C P}$.
Let us compare our results with those obtained in [56] for the simultaneous measurement of $\epsilon_{e \mu}, \epsilon_{e \tau}$ and $\epsilon_{e e}-\epsilon_{\mu \mu}$ and $\delta_{C P}$. As studied in [56], constraints on $\epsilon_{e \mu}, \epsilon_{e \tau}$ and $\epsilon_{e e}-\epsilon_{\mu \mu}$ are obtainted considering T2K, DUNE, and $\mathrm{NO} \nu \mathrm{A}$ experiments. Also in [68] the contraints on NSI parameters are obtained using DUNE, T2HK, and T2HKK experiments. Our results are consistant with those of $[56,68]$ when we consider only DUNE and T2HK and our results are an update to their analysis. Moreover, we have included the data of JUNO and considered the combined analysis of DUNE, T2HK, and JUNO. In conclusion, JUNO is going to play an important role in determining mass ordering and in the measurement of $\epsilon_{e e}-\epsilon_{\mu \mu}$ although it does not have a significant sensitivity to the determination of $\delta_{C P}$, octant of $\theta_{23}, \epsilon_{e \mu}$ and $\epsilon_{e \tau}$. 

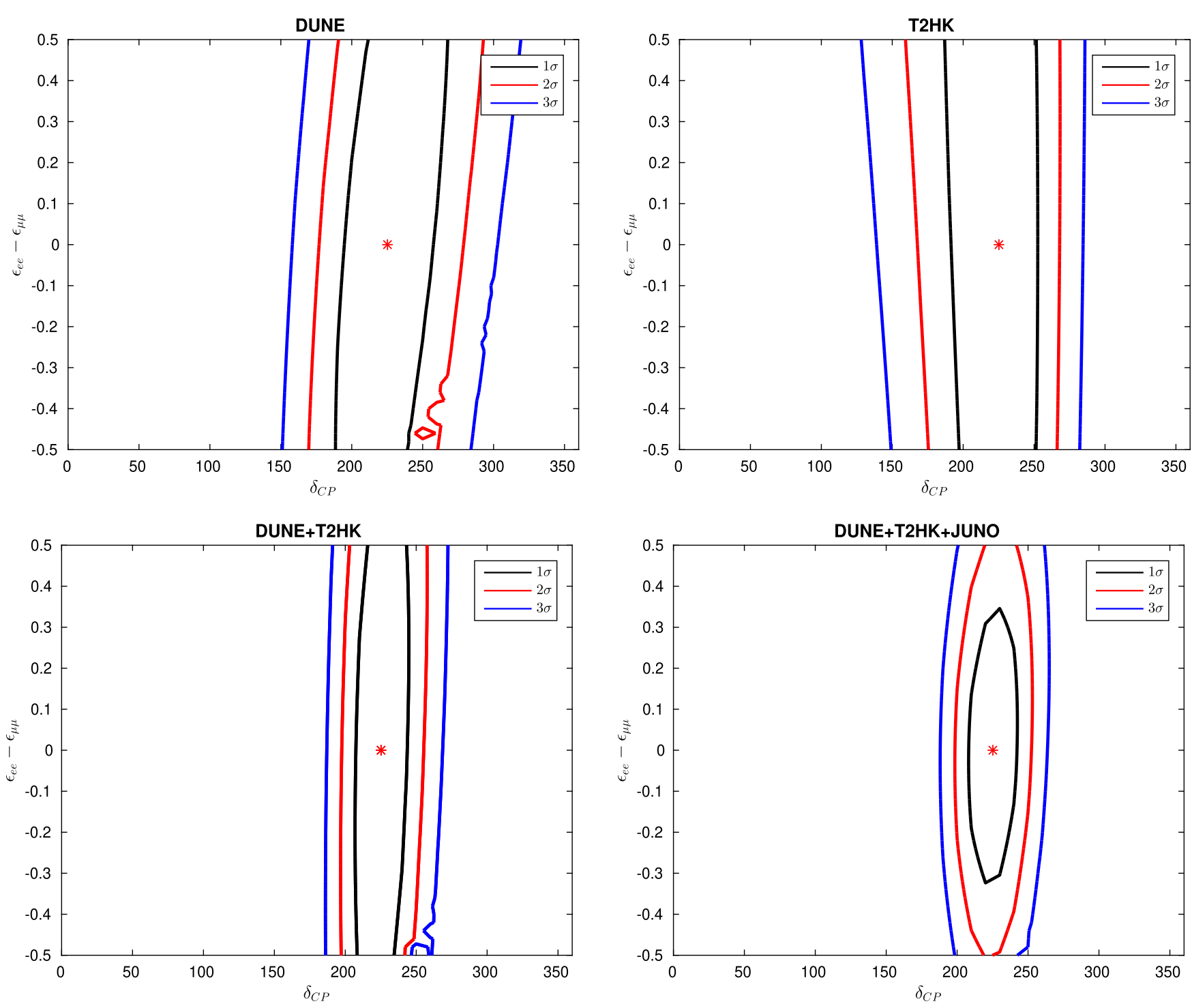

FIG. 8. $\epsilon_{e e}-\epsilon_{\mu \mu}$ vs. $\delta_{C P}$ plotted for plotted for T2HK, DUNE, T2HK + DUNE and T2HK + DUNE + JUNO. The red star shows the assumed true value for $\delta_{C P}$ and $\epsilon_{e e}-\epsilon_{\mu \mu}$. We have assumed 7 years of data taking for DUNE, 6 years of data taking for JUNO and 10 years of data taking for T2HK.

\section{SUMMARY}

In this paper, we explored the impact of NSI on future long-baseline neutrino experiments DUNE and T2HK in addition to the future reactor experiment JUNO. We investigated the potential of these experiments to the determination of the oscillation parameters in the presence of NSI. Moreover, we studied the possibility to constrain NSI parameters using these future neutrino experiments.

Having relatively low energy, the reactor experiment, JUNO is not sensitive to the matter effects. JUNO is going to provide the opportunity for the precise measurement of $\Delta m_{31}^{2}, \Delta m_{21}^{2}$ and $\theta_{12}$. We assumed the same NSI couplings for electron, up and down quarks and focused on the LMA solution.
We performed detailed numerical simulations using GLoBES and studied the measurements of standard neutrino oscillation parameters in the presence of NSI using combined data from future experiments, DUNE, T2HK, and JUNO. DUNE, T2HK, and their combination of data are sensitive to the determination of mass ordering at $2 \sigma$ C.L. while JUNO can determine the mass ordering at more than $3 \sigma$ as indicated in Fig. 2. DUNE and T2HK are not sensitive to the octant of $\theta_{23}$, separately while our results show that the combined data of these three experiments can determine the octant at about $3 \sigma$. As it is indicated in Fig. 4, DUNE and T2HK can determine $\delta_{C P}$ separately; However, the combination of data from DUNE, T2HK, and JUNO can increase the sensitivity to the determination of $\delta_{C P}$. As can be seen, the case of no $C P$-violation can be excluded at 
$2 \sigma$ using DUNE and T2HK. The combination of data of these two experiments can exclude it at $4 \sigma$ C.L. (Fig. 4).

While DUNE is sensitive to the determination of $\epsilon_{e \mu}$ and $\epsilon_{e \tau}$ and is going to constrain these parameters stronger than the current constraints, T2HK has weaker sensitivity to it. We have shown that considering the combination of DUNE, T2HK, and JUNO, can determine $\epsilon_{e \mu}$ and $\epsilon_{e \tau}$ much better than DUNE. Moreover, DUNE, T2HK and the combination of them are not sensitive to $\epsilon_{e e}-\epsilon_{\mu \mu}$. We have shown that considering the combination of DUNE, T2HK, and JUNO is sensitive to the determination of $\epsilon_{e e}-\epsilon_{\mu \mu}$ and can constrain it more stringent than the current constraints (Fig. 5). Overall, the combination of data from DUNE, T2HK, and JUNO can constrain $\epsilon_{e \mu}, \epsilon_{e \tau}$ and $\epsilon_{e e}-\epsilon_{\mu \mu}$ more stringent than the current constraints. The current constraints on $\epsilon_{e \mu}, \epsilon_{e \tau}$ and $\epsilon_{e e}-\epsilon_{\mu \mu}$ at $1 \sigma$ C.L. are 0.1, 0.25, and 0.3 , respectively [73], that are indicated by vertical green lines in the figures.

In addition, we have performed a combined analysis to study how combined data from DUNE, T2HK, and JUNO is sensitive to the simultaneous measurement of $\epsilon_{e \mu}$ and $\delta_{C P}$ (Fig. 6). In the same way, we studied how the combined analysis of DUNE, T2HK, and JUNO data can determine the simultaneous measurement of $\epsilon_{e \tau}$ and $\delta_{C P}$ as well as the simultaneous measurement of $\epsilon_{e e}-\epsilon_{\mu \mu}$ and $\delta_{C P}$ (Fig. 7 and Fig. 8). In more detail, DUNE and T2HK are sensitive to $\delta_{C P}$ while having a weak sensitivity to NSI parameters. Combining these experiments with JUNO improves slightly the sensitivity to $\delta_{C P}$ while increases the sensitivity to NSI parameters significantly.

Overall the combined analysis of future long-baseline experiments DUNE and T2HK, as well as the future reactor experiment JUNO is going to determine the standard oscillation parameters in the presence of NSI. Besides, the combination of these experiments can constrain the NSI parameters with higher precision.

\section{ACKNOWLEDGMENTS}

This project has received funding from the European Union's Horizon 2020 research and innovation programme under the Marie Skłodowska-Curie grant agreement No. 674896 and No. 690575. P. B thanks Iran Science Elites Federation Grant No. 11131.
[1] L. Wolfenstein, Phys. Rev. D 17, 2369 (1978).

[2] P. Minkowski, Phys. Lett. 67B, 421 (1977).

[3] T. Yanagida, Prog. Theor. Phys. 64, 1103 (1980).

[4] R. N. Mohapatra and G. Senjanovic, Phys. Rev. Lett. 44, 912 (1980).

[5] M. Gell-Mann, P. Ramond, and R. Slansky, Complex Spinors and Unified Theories, edited by D. Z. Freedman (North-Holland, Netherlands, 1979), https://inis.iaea.org/ search/search.aspx?orig_q=RN:11529248.

[6] J. Schechter and J. Valle, Phys. Rev. D 22, 2227 (1980).

[7] G. Lazarides, Q. Shafi, and C. Wetterich, Nucl. Phys. B181, 287 (1981).

[8] Y. Farzan and M. Tortola, Front. Phys. 6, 10 (2018).

[9] M. Gonzalez-Garcia, M. Maltoni, and T. Schwetz, J. High Energy Phys. 11 (2014) 052.

[10] F. Capozzi, G. Fogli, E. Lisi, A. Marrone, D. Montanino, and A. Palazzo, Phys. Rev. D 89, 093018 (2014).

[11] D. Forero, M. Tortola, and J. Valle, Phys. Rev. D 90, 093006 (2014).

[12] M. M. Guzzo, A. Masiero, and S. T. Petcov, Phys. Lett. B 260, 154 (1991).

[13] P. I. Krastev and S. T. Petcov, Phys. Lett. B 299, 99 (1993).

[14] O. G. Miranda, M. A. Tortola, and J. W. F. Valle, J. High Energy Phys. 10 (2006) 008.

[15] A. Bolaños, O. G. Miranda, A. Palazzo, M. A. Tortola, and J. W. F. Valle, Phys. Rev. D 79, 113012 (2009).

[16] A. Palazzo and J. W. F. Valle, Phys. Rev. D 80, 091301 (2009).
[17] F. J. Escrihuela, O. G. Miranda, M. A. Tortola, and J. W. F. Valle, Phys. Rev. D 80, 105009 (2009); 80, 129908(E) (2009).

[18] P. Bakhti and M. Rajaee, Phys. Rev. D 102, 035024 (2020).

[19] M. C. Gonzalez-Garcia, M. M. Guzzo, P. I. Krastev, H. Nunokawa, O. L. G. Peres, V. Pleitez, J. W. F. Valle, and R. Z. Funchal, Phys. Rev. Lett. 82, 3202 (1999).

[20] N. Fornengo, M. C. Gonzalez-Garcia, and J. W. F. Valle, J. High Energy Phys. 07 (2000) 006.

[21] N. Fornengo, M. Maltoni, R. Tomas, and J. W. F. Valle, Phys. Rev. D 65, 013010 (2001).

[22] P. Huber and J. W. F. Valle, Phys. Lett. B 523, 151 (2001).

[23] A. Friedland, C. Lunardini, and M. Maltoni, Phys. Rev. D 70, 111301 (2004).

[24] A. Friedland and C. Lunardini, Phys. Rev. D 72, 053009 (2005).

[25] O. Yasuda, Nucl. Phys. B, Proc. Suppl. 217, 220 (2011).

[26] M. C. Gonzalez-Garcia, M. Maltoni, and J. Salvado, J. High Energy Phys. 05 (2011) 075.

[27] A. Esmaili and A. Yu. Smirnov, J. High Energy Phys. 06 (2013) 026.

[28] S. Choubey and T. Ohlsson, Phys. Lett. B 739, 357 (2014).

[29] I. Mocioiu and W. Wright, Nucl. Phys. B893, 376 (2015).

[30] S. Fukasawa and O. Yasuda, arXiv:1503.08056.

[31] S. Choubey, A. Ghosh, T. Ohlsson, and D. Tiwari, J. High Energy Phys. 12 (2015) 01.

[32] A. Friedland and C. Lunardini, Phys. Rev. D 74, 033012 (2006). 
[33] M. Blennow, T. Ohlsson, and J. Skrotzki, Phys. Lett. B 660, 522 (2008).

[34] A. Esteban-Pretel, J. W. F. Valle, and P. Huber, Phys. Lett. B 668, 197 (2008).

[35] J. Kopp, P. A. N. Machado, and S. J. Parke, Phys. Rev. D 82, 113002 (2010).

[36] P. Coloma, A. Donini, J. Lopez-Pavon, and H. Minakata, J. High Energy Phys. 08 (2011) 036.

[37] A. Friedland and I. M. Shoemaker, arXiv:1207.6642.

[38] J. A. B. Coelho, T. Kafka, W. A. Mann, J. Schneps, and O. Altinok, Phys. Rev. D 86, 113015 (2012).

[39] P. Adamson et al. (MINOS Collaboration), Phys. Rev. D 88, 072011 (2013).

[40] P. Bakhti and Y. Farzan, J. High Energy Phys. 07 (2016) 109.

[41] S. Fukasawa, arXiv:1609.07240.

[42] S. Fukasawa, M. Ghosh, and O. Yasuda, arXiv:1609.04204.

[43] J. Salvado, O. Mena, S. Palomares-Ruiz, and N. Rius, J. High Energy Phys. 01 (2017) 141.

[44] K. J. Kelly, Phys. Rev. D 95, 115009 (2017).

[45] A. Chatterjee, P. Mehta, D. Choudhury, and R. Gandhi, Phys. Rev. D 93, 093017 (2016).

[46] P. S. B. Dev, K. S. Babu, P. B. Denton, P. A. N. Machado, C. A. Argüelles, J. L. Barrow, S. S. Chatterjee, M. C. Chen, A. de Gouvêa, B. Dutta et al., SciPost Phys. Proc. 2, 001 (2019).

[47] G. Mitsuka et al. (Super-Kamiokande Colloboration), Phys. Rev. D 84, 113008 (2011).

[48] M. C. Gonzalez-Garcia and M. Maltoni, Phys. Rev. D 70, 033010 (2004).

[49] P. Bakhti, A. N. Khan, and W. Wang, J. Phys. G 44, 125001 (2017).

[50] A. N. Khan, D. W. McKay, and F. Tahir, Phys. Rev. D 88, 113006 (2013).

[51] A. N. Khan, D. W. McKay, and F. Tahir, Phys. Rev. D 90, 053008 (2014).

[52] A. N. Khan, Phys. Rev. D 93, 093019 (2016).

[53] I. Girardi, D. Meloni, and S. T. Petcov, Nucl. Phys. B886, 31 (2014).

[54] I. Girardi and D. Meloni, Phys. Rev. D 90, 073011.

[55] A. de Gouvea and K. J. Kelly, Nucl. Phys. B908, 318 (2016).

[56] J. Liao, D. Marfatia, and K. Whisnant, Phys. Rev. D 93, 093016 (2016).

[57] P. Coloma, J. High Energy Phys. 03 (2016) 016.

[58] P. Coloma and T. Schwetz, Phys. Rev. D 95, 079903 (2017).

[59] M. Masud and P. Mehta, Phys. Rev. D 94, 053007 (2016).

[60] F. P. An et al. (Daya Bay Collaboration), Phys. Rev. D 95, 072006 (2017).
[61] S. H. Seo et al. (RENO Collaboration), Phys. Rev. D 98, 012002 (2018).

[62] A. Gando et al. (KamLAND Collaboration), Phys. Rev. D 83, 052002 (2011).

[63] K. Abe et al. (T2K Collaboration), Phys. Rev. Lett. 107, 041801 (2011).

[64] H. T. J. Steiger (JUNO Collaboration), arXiv:1912.02038.

[65] A. de Gouvea and K. J. Kelly, Nucl. Phys. B908, 318 (2016).

[66] A. Giarnetti and D. Meloni, arXiv:2005.10272.

[67] KEK Preprint 2016-21 and ICRR-Report-701-2016-1, https://libextopc.kek.jp/preprints/PDF/2016/1627/1627021 .pdf.

[68] J. Liao, D. Marfatia, and K. Whisnant, J. High Energy Phys. 01 (2017) 071.

[69] R. Adhikari, S. Chakraborty, A. Dasgupta, and S. Roy, Phys. Rev. D 86, 073010 (2012).

[70] I. Girardi, D. Meloni, and S. T. Petcov, Nucl. Phys. B886, 31 (2014).

[71] B. Pontecorvo, Zh. Eksp. Teor. Fiz. 53, 1717 (1967).

[72] Z. Maki, M. Nakagawa, and S. Sakata, Prog. Theor. Phys. 28, 870 (1962).

[73] I. Esteban, M. C. Gonzalez-Garcia, and M. Maltoni, J. High Energy Phys. 06 (2019) 055.

[74] P. Coloma, M. C. Gonzalez-Garcia, M. Maltoni, and T. Schwetz, Phys. Rev. D 96, 115007 (2017).

[75] I. Esteban, M. C. Gonzalez-Garcia, and M. Maltoni, arXiv:2004.04745.

[76] J. Kopp, M. Lindner, T. Ota, and J. Sato, Phys. Rev. D 77, 013007 (2008).

[77] I. Esteban, M. C. Gonzalez-Garcia, M. Maltoni, T. Schwetz, and A. Zhou, J. High Energy Phys. 09 (2020) 178.

[78] R. Acciarri et al. (DUNE Collaboration), arXiv:1512 .06148 .

[79] Hyper-Kamiokande Collaboration, Reports No. KEK-PREPRINT-2016-21, No. ICRR-REPORT-701-2016-1.

[80] P. Bakhti and A. Y. Smirnov, Phys. Rev. D 101, 123031 (2020).

[81] Y. Itow et al. (T2K Colloboration), arXiv:hep-ex/0106019.

[82] P. Bakhti and Y. Farzan, J. High Energy Phys. 10 (2013) 200.

[83] P. Bakhti and Y. Farzan, J. High Energy Phys. 07 (2014) 064.

[84] P. Huber, M. Lindner, and W. Winter, Comput. Phys. Commun. 167, 195 (2005).

[85] P. Huber, J. Kopp, M. Lindner, M. Rolinec, and W. Winter, Comput. Phys. Commun. 177, 432 (2007). 